\title{
Control and Stabilization of High-Order KdV Equation Posed on the Periodic Domain
}

\author{
ZHAO Xiangqing ${ }^{1,2}$ and BAI Meng ${ }^{3, *}$ \\ ${ }^{1}$ Department of Mathematics, Zhejiang Ocean University, Zhoushan 316000, China. \\ 2 Key Laboratory of Oceanographic Big Data Mining \& Application of Zhejiang \\ Province, Zhoushan 316000, China. \\ 3 School of Mathematics and Statistics Sciences, Zhaoqing University, \\ Zhaoqing 526061, China.
}

Received 20 July 2017; Accepted 30 November 2017

\begin{abstract}
In this paper, we study exact controllability and feedback stabilization for the distributed parameter control system described by high-order KdV equation posed on a periodic domain $\mathbb{T}$ with an internal control acting on an arbitrary small nonempty subdomain $\omega$ of $\mathbb{T}$. On one hand, we show that the distributed parameter control system is locally exactly controllable with the help of Bourgain smoothing effect; on the other hand, we prove that the feedback system is locally exponentially stable with an arbitrarily large decay rate when Slemrod's feedback input is chosen.
\end{abstract}

AMS Subject Classifications: 93B05, 93D15, 35Q53

Chinese Library Classifications: O175.2, O175.4, O29

Key Words: High-order KdV equation; Bourgain smoothing property; exact controllability; Slemrod's feedback law; exponential stabilizability.

\section{Introduction}

In this paper, we will investigate the following higher-order dispersive equation posed on the periodic domain $\mathbb{T}$ (a unit circle in the plane) from the control point of view:

$$
\partial_{t} u+(-1)^{l+1} \partial_{x}^{2 l+1} u+u \partial_{x} u=f, \quad x \in \mathbb{\Gamma}, t \in \mathbb{R},
$$

${ }^{*}$ Corresponding author. Email addresses: zhao-xiangqing@163.com (X.Q. Zhao), baimengclare@qq.com (M. Bai) 
where $f$ is the control input supported in a given open set $\omega \subset \mathbb{T}$. The assumption on the periodic domain is equivalent to impose the periodic boundary conditions over the interval $(0,2 \pi)$ :

$$
\partial_{x}^{n} u(0, t)=\partial_{x}^{n} u(2 \pi, t), \quad n=0,1, \cdots, 2 l .
$$

The following two fundamental control theory problems will be discussed:

Exact controllability: For the given initial state $u_{0}$ and terminal state $u_{1}$ belong in a certain space, can one find an appropriate control input $f$ such that equation (1.1) admits a solution $u$ which satisfies

$$
\left.u\right|_{t=0}=u_{0},\left.\quad u\right|_{t=T}=u_{1} ?
$$

Feedback stabilization: Is there a feedback control law: $f=K u$ such that the resulting closedloop system

$$
\partial_{t} u+(-1)^{l+1} \partial_{x}^{2 l+1} u+u \partial_{x} u=K u, \quad x \in \mathbb{T}, t \in \mathbb{R}
$$

is exponentially stable as $t \rightarrow \infty$ ?

Since for the solution of (1.1) satisfies

$$
\frac{\mathrm{d}}{\mathrm{d} t} \int_{\mathbb{T}} u(x, t) \mathrm{d} x=\int_{\mathbb{T}} f(x, t) \mathrm{d} x,
$$

the mass will be conserved provided that

$$
\int_{\mathbb{T}} f(x, t) \mathrm{d} x=0 .
$$

For the purpose of mass conservation, the control input as follows is chosen (see [5]):

$$
f(x, t)=[G h](x, t):=g(x)\left(h(x, t)-\int_{\mathbb{T}} g(y) h(y, t) \mathrm{d} y\right)
$$

where $g(x)$ is a given nonnegative smooth function such that $\{g>0\}=\omega \subset \mathbb{T}$ and

$$
2 \pi[g]=\int_{\mathbb{T}} g(x) \mathrm{d} x=1 .
$$

With $h$ as a new control input, the resulting control system turns to be

$$
\partial_{t} u+(-1)^{l+1} \partial_{x}^{2 l+1} u+u \partial_{x} u=G h, \quad x \in \mathbb{T}, t \in \mathbb{R} .
$$

We state the main results as follows:

Theorem 1.1 (Exact controllability). Let $T>0$ and $s \geq s_{0}$ (see Lemma 3.2) be given. Then there exists a $\delta>0$ such that for any $u_{0}, u_{1} \in H^{s}(\mathbb{T})$ with $\left[u_{0}\right]=\left[u_{1}\right]$ and

$$
\left\|u_{0}\right\|_{H^{s}(\mathbb{T})} \leq \delta, \quad\left\|u_{1}\right\|_{H^{s}(\mathbb{T})} \leq \delta .
$$


one can find a control function $h \in L^{2}\left([0, T] ; H^{s}(\mathbb{T})\right)$ such that the system (1.3) has a solution $u \in C\left([0, T] ; H^{s}(\mathbb{T})\right)$ satisfying

$$
\left.u\right|_{t=0}=u_{0},\left.\quad u\right|_{t=T}=u_{1} .
$$

Theorem 1.2 (Feedback Stabilizability). Let $s \geq s_{0}$ and $\lambda>0$ be given. If one chooses the Slemrod's feedback control $K_{\lambda}$ (see Section 2) in the system (1.3), then the resulting close-loop system

$$
\begin{cases}\partial_{t} u+(-1)^{l+1} \partial_{x}^{2 l+1} u+u \partial_{x} u=G K_{\lambda} u, & x \in \mathbb{T}, t \in \mathbb{R}, \\ u(x, 0)=u_{0}(x), & x \in \mathbb{T}\end{cases}
$$

is locally exponentially stable in the space $H^{s}(\mathbb{T})$ : there exists $\delta>0$ such that for any $u_{0} \in H^{s}(\mathbb{T})$ with $\left\|u_{0}\right\|_{H^{s}(\mathbb{T})}<\delta$, the corresponding solution $u$ of (1.4) satisfies

$$
\left\|u(\cdot, t)-\left[u_{0}\right]\right\|_{H^{s}(\mathbb{T})} \leq C e^{-\lambda t}\left\|u_{0}-\left[u_{0}\right]\right\|_{H^{s}(\mathbb{T})},
$$

for any $t>0$.

When $l=1$, the dispersive equation

$$
\partial_{t} u+(-1)^{l+1} \partial_{x}^{2 l+1} u+u \partial_{x} u=0,
$$

became the famous $\mathrm{KdV}$ equation, therefor people often call (1.5) as high-order KdV equation [1,2]. More information on higher-order dispersive equations can be found in [3]. The periodic exact controllability and exponentially stability of KdV equation and Kawahara equation are investigated by $[4-6]$ and $[7,8]$ respectively.

The Cauchy problem of the general dispersive equation (1.5) has been shown wellposed recently by Bourgain method (see $[1,2,9]$ ). The subtle Bourgain smoothing effect established in $[1,2,9]$ will play an indispensable role in the proofs of exactly controllable and exponentially stability in this paper. Results on initial-boundary value problem for (1.5) see [10-14].

Since it is time-reversible for dispersive equation, we assume $t>0$ in the following sections.

The paper is organized as follows: In Section 2, we study the associated linearized system. We obtain the controllability of the linear open loop system in the space $H^{s}(\mathbb{T})$ for any $s \in \mathbb{R}$ through solving a moment problem. Then the exponentially stabilizable with arbitrarily large decay rate is proved when the Slemrod's feedback control input is chosen. In Section 3, aided by Bourgain smoothing properties of linear equation, we show the nonlinear system is locally exactly controllable in the space $H^{s}(\mathbb{T})$ for any $s \geq s_{0}$ by Banach fixed point theorem. In Section 4, the nonlinear feedback system is first shown to be globally well-posed in the space $H^{s}(\mathbb{T})$ for any $s \geq s_{0}$ and then it is shown to locally exponentially stabilizable with arbitrarily large decay rate. 


\section{Linear system}

Consideration is first given to the associated linear open loop control system

$$
\begin{aligned}
& \partial_{t} v+(-1)^{l+1} \partial_{x}^{2 l+1} v=G h, \\
& v(x, 0)=v_{0}(x), \quad x \in \mathbb{T}, t \in \mathbb{R}
\end{aligned}
$$

where the operator $G$ and control input $h=h(x, t)$ are defined in Section 1.

Let $A$ denote the operator

$$
A w=(-1)^{l+1} \frac{d^{2 l+1}}{\mathrm{~d} x^{2 l+1}} w
$$

with the domain $\mathcal{D}(A)=H^{2 l+1}(\mathbb{T})$. It generates a strongly continuous group $W(t)$ on the space $L^{2}(\mathbb{T})$ and its eigenfunctions are simply the orthonormal Fourier basis functions in $L^{2}(\mathbb{T})$,

$$
\phi_{k}(x)=\frac{1}{\sqrt{2 \pi}} e^{i k x}, \quad k=0, \pm 1, \cdots .
$$

The corresponding eigenvalue of $\phi_{k}$ is

$$
\lambda_{k}=-k^{2 l+1} i, \quad k=0, \pm 1, \cdots .
$$

For any $l \in \mathbb{Z}$, let

$$
m(l)=\#\left\{k \in \mathbb{Z} ; \quad \lambda_{k}=\lambda_{l}\right\} .
$$

Then $m(l) \leq 2 l+1$ for any $l$ and $m(l)=1$ if $l$ is large enough. Moreover,

$$
\lim _{|k| \rightarrow \infty}\left|\lambda_{k}-\lambda_{k+1}\right|=\infty
$$

The solution $v$ of the system (2.1) can be expressed in the form

$$
v(x, t)=\sum_{k=-\infty}^{\infty}\left(e^{\lambda_{k} t} v_{0, k}+\int_{0}^{t} e^{\lambda_{k}(t-\tau)} G_{k}[h](\tau) \mathrm{d} \tau\right) \phi_{k}(x),
$$

where $v_{0, k}$ and $G_{k}[h]$ are the Fourier coefficients of $v_{0}$ and $G[h]$, respectively,

$$
v_{0, k}=\left(v_{0}, \phi_{k}\right)_{L^{2}(\mathbb{T})}, \quad G_{k}[h]=\left(G h, \phi_{k}\right)_{L^{2}(\mathbb{T})}=\left(h, G \phi_{k}\right)_{L^{2}(\mathbb{T})}
$$

for $k=0, \pm 1, \pm 2, \cdots$. Furthermore, for given $s \in \mathbb{R}$, if $v_{0} \in H^{s}(\mathbb{T})$ and $h \in L^{2}\left(0, T ; H^{s}(\mathbb{T})\right)$, the function given by (2.2) belongs to the space $C\left([0, T] ; H^{s}(\mathbb{T})\right)$.

We have the following exact controllability result for the open loop control system (2.1). 
Theorem 2.1. Let $T>0$ and $s \in \mathbb{R}$ be given. There exists a bounded linear operator

$$
\Phi: H^{s}(\mathbb{T}) \times H^{s}(\mathbb{T}) \rightarrow L^{2}\left(0, T ; H^{s}(\mathbb{T})\right)
$$

such that for any $v_{0}, v_{1} \in H^{s}(\mathbb{T})$, if one chooses $h=\Phi\left(v_{0}, v_{T}\right)$ in (2.1), then the system (2.1) admits a solution $v \in C\left([0, T] ; H^{s}(\mathbb{T})\right)$ satisfying

$$
\left.v\right|_{t=0}=v_{0},\left.\quad v\right|_{t=T}=v_{1} .
$$

In the sequel we will denote by $C$ numerical constant which maybe different from line to line. Moreover, let

$$
\begin{aligned}
& \|f\|_{s}:=\|f\|_{H^{s}(\mathbb{T})} \quad \text { for any } f \in H^{s}(\mathbb{T}) \\
& \|f\|:=\|f\|_{0} .
\end{aligned}
$$

Proof. For give $v_{0}, v_{1} \in H^{s}(\mathbb{T})$, we need to find $h \in L^{2}\left(0, T ; H^{s}(\mathbb{T})\right)$ such

$$
v_{1}(x)=\sum_{k=-\infty}^{\infty}\left(e^{\lambda_{k} T} v_{0, k}+\int_{0}^{T} e^{\lambda_{k}(T-\tau)} G_{k}[h](\tau) \mathrm{d} \tau\right) \phi_{k}(x)
$$

or

$$
\sum_{k=-\infty}^{\infty}\left(v_{1, k}-e^{-\lambda_{k} T} v_{0, k}\right) \phi_{k}=\sum_{k=-\infty}^{\infty} \int_{0}^{T} e^{-\lambda_{k} \tau} G_{k}[h](\tau) \mathrm{d} \tau \phi_{k}(x)
$$

which is equivalent to the moment equation:

$$
v_{1, k}-e^{-\lambda_{k} T} v_{0, k}=\int_{0}^{T} e^{-\lambda_{k} \tau} G_{k}[h](\tau) \mathrm{d} \tau
$$

for $k= \pm 1, \pm 2, \cdots$.

If we define $p_{k}=e^{\lambda_{k} t}$, then $\mathcal{P} \equiv\left\{p_{k} \mid-\infty<k<\infty\right\}$ will form a Riesz basis for its closed span $\mathcal{P}_{T}$ in $L^{2}(0, T)$. We let $\mathcal{Q} \equiv\left\{q_{k} \mid-\infty<k<\infty\right\}$ be the unique dual Riesz basis for $\mathcal{P}$ in $\mathcal{P}_{T}$ such that

$$
\int_{0}^{T} q_{j}(t) \overline{p_{k}(t)} \mathrm{d} t=\delta_{j k,} \quad-\infty<j, k<\infty .
$$

We take the control $h$ in (2.3) to have the form

$$
h(x, t)=\sum_{j=-\infty}^{\infty} h_{j} q_{j}(t)\left(G \phi_{j}\right)(x),
$$

where the coefficients $h_{j}$ are to be determined so that, among other things, the series (2.5) is appropriately convergent. Substituting (2.5) into (2.3) yields, using the biorthogonality (2.4), that

$$
v_{1, k}-e^{-\lambda_{k} T} v_{0, k}=\sum_{-\infty}^{\infty} h_{j} \int_{0}^{T} e^{-\lambda_{k} t} q_{j}(t) \int_{\mathbb{T}} G\left(G \phi_{j}\right)(x) \overline{\phi_{k}(x)} \mathrm{d} x \mathrm{~d} t=h_{k} \int_{\mathbb{T}} G\left(G \phi_{k}\right)(x) \overline{\phi_{k}(x)} \mathrm{d} x \mathrm{~d} t,
$$


for $-\infty<k<\infty$. As $G$ is a self-adjoint operator in $L^{2}(\mathbb{T})$,

$$
\int_{\mathbb{T}} G\left(G \phi_{k}\right)(x) \overline{\phi_{k}(x)} \mathrm{d} x \mathrm{~d} t=\left\|G \phi_{k}\right\|^{2}, \quad-\infty<k<\infty .
$$

We have

$$
\begin{aligned}
\left\|G \phi_{k}\right\|^{2} & =\int_{\mathbb{T}}\left|g(x)\left(\phi_{k}(x)-\int_{\mathbb{T}} g(s) \phi_{k}(s) \mathrm{d} s\right)\right|^{2} \mathrm{~d} x \\
& =\frac{1}{4 \pi^{2}} \int_{\mathbb{T}} g^{2}(x) \mathrm{d} x-2\left|\int_{\mathbb{T}} g(x) \phi_{k}(x) \mathrm{d} x\right|^{2}+\int_{\mathbb{T}} g^{2}(x) \mathrm{d} x\left|\int_{\mathbb{T}} g(x) \phi_{k}(x) \mathrm{d} x\right|^{2}=: \beta_{k} .
\end{aligned}
$$

It is easy to see that $\beta_{0}=0$ and $\beta_{k} \neq 0$ if $k \neq 0$. Moreover, the familiar Lebesgue lemma together with the second identity above shows that

$$
\lim _{k \rightarrow \infty} \beta_{k}=\int_{\mathbb{T}} g^{2}(x) \mathrm{d} x \neq 0 .
$$

It follows that there is a $\delta>0$ such that

$$
\beta_{k}>\delta, \quad \text { for } k \neq 0 \text {. }
$$

Setting $h_{0}=0$ and

$$
h_{k}=\frac{e^{-\lambda_{k} T} v_{1, k}-v_{0, k}}{\beta_{k}}, \quad k \neq 0,
$$

It remains to show that $h$ defined by $(2.5)$ and $(2.7)$ is in $L^{2}\left([0, T] ; H^{s}(\mathbb{T})\right)$ provided that $v_{0}, v_{1} \in H^{s}(\mathbb{T})$. To this end, let us write

$$
G \phi_{k}(x)=\sum_{k=-\infty}^{\infty} a_{j k} \phi_{j}(x)
$$

where

$$
a_{j k}=\int_{\mathbb{T}} G \phi_{j}(x) \overline{\phi_{k}(x)} \mathrm{d} x=\left(G \phi_{j}(x), \phi_{k}(x)\right)_{L^{2}(\mathbb{T}),} \quad-\infty<j, k<\infty .
$$

Thus

$$
h(x, t)=\sum_{j=-\infty}^{\infty} \sum_{k=-\infty}^{\infty} h_{j} a_{j k} q_{j}(t) \phi_{k}(x)
$$

and

$$
\begin{aligned}
& \|h\|_{L^{2}\left([0, T] ; H^{s}(\mathbb{T})\right)}^{2} \\
= & \int_{0}^{T} \sum_{k=-\infty}^{\infty}\langle k\rangle^{2 s}\left|\sum_{j=-\infty}^{\infty} h_{j} a_{j k} q_{j}(t)\right|^{2} \mathrm{~d} t=\sum_{k=-\infty}^{\infty}\langle k\rangle^{2 s} \int_{0}^{T}\left|\sum_{j=-\infty}^{\infty} h_{j} a_{j k} q_{j}(t)\right|^{2} \mathrm{~d} t
\end{aligned}
$$




$$
\leq C \sum_{k=-\infty}^{\infty}\langle k\rangle^{2 s} \sum_{j=-\infty}^{\infty}\left|h_{j}\right|^{2}\left|a_{j k}\right|^{2}=C \sum_{j=-\infty}^{\infty}\left|h_{j}\right|^{2} \sum_{k=-\infty}^{\infty}\langle k\rangle^{2 s}\left|a_{j k}\right|^{2},
$$

where the constant $C$ comes from the Riesz basis property of $\mathcal{Q}$ in $\mathcal{P}_{T}$. However

$$
\begin{aligned}
\left|a_{i j}\right| & =\left(G \phi_{j}, \phi_{k}\right)_{L^{2}(\mathbb{T})}=\left|\left(g \phi_{j}, \phi_{k}\right)_{L^{2}(\mathbb{T})}-\left(g, \phi_{j}\right)_{L^{2}(\mathbb{T})}\left(g, \phi_{k}\right)_{L^{2}(\mathbb{T})}\right| \\
& =\left|\sum_{m=-\infty}^{\infty} g_{m}\left(\phi_{m} \phi_{j}, \phi_{k}\right)_{L^{2}(\mathbb{T})}-\left(\sum_{m=-\infty}^{\infty} g_{m}\left(\phi_{m}, \phi_{j}\right)_{L^{2}(\mathbb{T})}\right)\left(\sum_{m=-\infty}^{\infty} g_{m}\left(\phi_{m}, \phi_{k}\right)_{L^{2}(\mathbb{T})}\right)\right| \\
& =\left|\frac{1}{2 \pi} g_{k-j}-g_{j} g_{k}\right|=\frac{1}{2 \pi}\left|g_{k-j}\right|+\left|g_{j}\right|\left|g_{k}\right|,
\end{aligned}
$$

where

$$
g(x)=\sum_{j=-\infty}^{\infty} g_{m} \phi_{m}(x)
$$

Hence

$$
\left|a_{j k}\right|^{2} \leq C\left(\left|g_{k-j}\right|^{2}+\left|g_{k}\right|^{2}\left|g_{j}\right|^{2}\right)
$$

and

$$
\begin{aligned}
\sum_{k=-\infty}^{\infty}\langle k\rangle^{2 s}\left|a_{j k}\right|^{2} & \leq C \sum_{k=-\infty}^{\infty}\langle k\rangle^{2 s}\left|g_{k-j}\right|^{2}+C \sum_{k=-\infty}^{\infty}\langle k\rangle^{2 s}\left|g_{j}\right|^{2}\left|g_{k}\right|^{2} \\
& =C \sum_{k=-\infty}^{\infty}\langle k+j\rangle^{2 s}\left|g_{k}\right|^{2}+C\left|g_{j}\right|^{2} \sum_{k=-\infty}^{\infty}\langle k\rangle^{2 s}\left|g_{k}\right|^{2} .
\end{aligned}
$$

Thus, in the case of $s \geq 0$,

$$
\begin{aligned}
\sum_{k=-\infty}^{\infty}\langle k\rangle^{2 s}\left|a_{j k}\right|^{2} & \leq C \sum_{k=-\infty}^{\infty}\langle k\rangle^{2 s}\langle j\rangle^{2 s}\left|g_{k}\right|^{2}+C\left|g_{j}\right|^{2} \sum_{k=-\infty}^{\infty}\langle k\rangle^{2 s}\left|g_{k}\right|^{2} \\
& =C\left(\langle j\rangle^{2 s}+\left|g_{j}\right|^{2}\right)\|g\|_{s}^{2} .
\end{aligned}
$$

We have, according to (2.7), that

$$
\begin{aligned}
\|h\|_{L^{2}\left([0, T] ; H^{s}(\mathbb{T})\right)}^{2} & \leq C\left[\sum_{j=-\infty}^{\infty}\left(\langle j\rangle^{2 s}+\left|g_{j}\right|^{2}\right)\left|h_{j}\right|^{2}\right]\|g\|_{s}^{2} \\
& \leq C\left[\sum_{j=-\infty}^{\infty}\left(\langle j\rangle^{2 s}+\left|g_{j}\right|^{2}\right) \frac{\left|e^{\lambda_{j} T} v_{j, 1}-v_{j, 0}\right|^{2}}{\beta_{j}^{2}}\right]\|g\|_{s}^{2} \\
& \leq C \max _{j \neq 0}\left|\beta_{j}\right|^{-2}\left(1+\|g\|_{0}^{2}\right) \sum_{j=-\infty}^{\infty}\langle j\rangle^{2 s}\left(\left|v_{j, 1}\right|^{2}+\left|v_{j, 0}\right|^{2}\right) \\
& \leq C \max _{j \neq 0} \frac{1}{\left|\beta_{j}\right|^{2}}\left(1+\|g\|_{0}^{2}\right)\|g\|_{s}^{2}\left(\left\|v_{1}\right\|_{s}^{2}+\|\left. v_{0}\right|_{s} ^{2}\right) .
\end{aligned}
$$


In the case of $s<0$, as for any $-\infty<k, j<\infty$,

$$
\begin{aligned}
& \langle j\rangle^{-2 s}\langle k+j\rangle^{2 s} \leq\langle k\rangle^{-2 s}, \quad\langle j\rangle^{-2 s}\left|g_{j}\right|^{2} \leq\|g\|_{-s}^{2}, \\
& \sum_{k=-\infty}^{\infty}\langle j\rangle^{-2 s}\langle k\rangle^{2 s}\left|a_{j k}\right|^{2} \leq C \sum_{k=-\infty}^{\infty}\langle j\rangle^{-2 s}\langle k+j\rangle^{2 s}\left|g_{k}\right|^{2}+C\langle j\rangle^{-2 s}\left|g_{j}\right|^{2} \sum_{k=-\infty}^{\infty}\langle k\rangle^{2 s}\left|g_{k}\right|^{2} \\
\leq & C\left(1+\|g\|_{s}^{2}\right)\|g\|_{-s}^{2}
\end{aligned}
$$

and therefore

$$
\begin{aligned}
\|h\|_{L^{2}\left([0, T] ; H^{s}(\mathbb{T})\right)}^{2} & \leq C \sum_{j=-\infty}^{\infty}\left|h_{j}\right|^{2} \sum_{k=-\infty}^{\infty}\langle k\rangle^{2 s}\left|a_{j k}\right|^{2} \\
& \leq C \sum_{j=-\infty}^{\infty}\langle j\rangle^{2 s}\left|h_{j}\right|^{2} \sum_{k=-\infty}^{\infty}\langle j\rangle^{-2 s}\langle k\rangle^{2 s}\left|a_{j k}\right|^{2} \\
& \leq C\left(1+\|g\|_{s}^{2}\right)\|g\|_{-s}^{2} \sum_{j=-\infty}^{\infty}\langle j\rangle^{2 s}\left|h_{j}\right|^{2} \\
& \leq C \max _{j \neq 0} \frac{1}{\left|\beta_{j}\right|^{2}}\left(1+\|g\|_{s}^{2}\right)\|g\|_{-s}^{2}\left(\left\|v_{1}\right\|_{s}^{2}+\|\left. v_{0}\right|_{s} ^{2}\right) .
\end{aligned}
$$

Now we turn to consider feedback stabilization problem of the linear system (2.1). According to [4,7], it is possible to establish the exponential stability with decay rate as large as one desires for the resulting closed-loop system if the Selmord feedback law is chosen. For any $\lambda>0$, define

$$
L_{\lambda} \phi=\int_{0}^{1} e^{-2 \lambda \tau} W(-\tau) G G^{*} W^{*}(-\tau) \phi \mathrm{d} \tau
$$

for any $\phi \in H^{s}(\mathbb{T})$. Then, we have

Lemma 2.1. For any $s \geq 0$, the operator $L_{\lambda}$ is an isomorphism from $H^{s}(\mathbb{T})$ onto $H^{s}(\mathbb{T})$ for all $s \geq 0$.

Proof. See Lemma 2.4 in [4].

According to Lemma 2.1, $L_{\lambda}$ has bounded inverse in $H^{s}(\mathbb{T})$. Taking the control function $h(x, t)=-G^{*} L_{\lambda}^{-1} v(x, t)$, we obtain the following closed-loop system:

$$
\partial_{t} v+(-1)^{l+1} \partial_{x}^{2 l+1} v=-K_{\lambda} v, \quad v(x, 0)=v_{0}(x), \quad x \in \mathbb{T}
$$

with the feedback control law

$$
K_{\lambda} v(x, t) \equiv-G G^{*} L_{\lambda}^{-1} v(x, t) .
$$


Proposition 2.1. Let $s \geq 0$ and $\lambda>0$ be given. Then for any $v_{0} \in H^{s}(\mathbb{T})$, the system (2.9) admits a unique solution $v \in C\left([0, T] ; H^{s}(\mathbb{T})\right)$. Moreover there exist positive constants $M_{s}$ depending only on s such that

$$
\|v(\cdot, t)\|_{s} \leq M_{s} e^{-\lambda t}\left\|v_{0}\right\|_{s}
$$

for any $t>0$.

Proof. The existence of the solution $v$ follows from the standard semigroup theory [15]. The decay estimate (2.10) can be proved by interpolation:

The case of $s=0$ follows from [16].

For $s=2 l+1$, let $w=v_{t}$. Then $w$ solves

$$
\partial_{t} w+(-1)^{l+1} \partial_{x}^{2 l+1} w=-K_{\lambda} w, \quad w(x, 0)=w_{0}(x), \quad x \in \mathbb{T}
$$

where $w_{0}(x)=(-1)^{l} v_{0}^{(2 l+1)}(x)-K_{\lambda} v_{0}(x)$. Thus

$$
\|w(\cdot, t)\|=\left\|v_{t}(\cdot, t)\right\| \leq C e^{-\lambda t}\left\|w_{0}\right\|
$$

for any $t \geq 0$. It then follows from

$$
w=(-1)^{l} \partial_{x}^{2 l+1} v-K_{\lambda} v
$$

that

$$
\|v(\cdot, t)\|_{2 l+1} \leq e^{-\lambda t}\left\|v_{0}\right\|_{2 l+1}
$$

for any $t \geq 0$.

The case of $0<s<2 l+1$ follows by interpolation. The other cases of $s$ can be proved similarly.

\section{Exact controllability}

In this section, we study the exact controllability for the open loop nonlinear control system:

$$
\partial_{t} u+(-1)^{l+1} \partial_{x}^{2 l+1} u+u \partial_{x} u=G h, \quad u(x, 0)=u_{0}(x), \quad x \in \mathbb{T}, \quad t>0 .
$$

To apply the Bourgain smoothing effect, some technical preparations are needed. For given $b, s \in \mathbb{R}$, and a function $u: \mathbb{T} \times \mathbb{R} \rightarrow \mathbb{R}$, define the quantities

$$
\|u\|_{X_{b, s}}:=\left(\sum_{k=-\infty}^{\infty} \int_{\mathbb{R}}\langle k\rangle^{2 s}\langle\tau-p(k)\rangle^{2 b}|\widehat{u}(k, \tau)|^{2} \mathrm{~d} \tau\right)^{\frac{1}{2}},
$$




$$
\|u\|_{\gamma_{b, s}}:=\left(\sum_{k=-\infty}^{\infty}\left(\int_{\mathbb{R}}\langle k\rangle^{s}\langle\tau-p(k)\rangle^{b}|\widehat{u}(k, \tau)| \mathrm{d} \tau\right)^{2}\right)^{\frac{1}{2}}
$$

where $\widehat{u}(k, \tau)$ denotes the Fourier transform of $u$ with respect to the space variable $x$ and the time variable $t,\langle\cdot\rangle=\sqrt{1+|\cdot|^{2}}$ and $p(k)=-k^{2 l+1}$. Then Bourgain space $X_{b, s}\left(\operatorname{resp} . Y_{b, s}\right)$ associated to the higher-order $\mathrm{KdV}$ equation on $\mathbb{T}$ is the completion of the space $\mathcal{S}(\mathbb{T} \times \mathbb{R})$ under the norm $\|u\|_{X_{b, s}}$ (resp. $\|u\|_{Y_{b, s}}$ ).

For given $b, s \in \mathbb{R}$, let

$$
Z_{b, s}=X_{b, s} \cap Y_{b-\frac{1}{2}, s}
$$

be endowed with the norm

$$
\|u\|_{Z_{b, s}}=\|u\|_{X_{b, s}}+\|u\|_{Y_{b-\frac{1}{2}, s}}
$$

For a given interval $I$, let $X_{b, s}(I)$ (resp. $\left.Z_{b, s}(I)\right)$ be the restriction space of $X_{b, s}$ to the interval $I$ with the norm

$$
\begin{aligned}
& \|u\|_{X_{b, s}(I)}=\inf \left\{\|\widetilde{u}\|_{X_{b, s}(I)} \mid \widetilde{u}=u \text { on } \mathbb{T} \times I\right\} \\
& \left(\text { resp. }\|u\|_{Z_{b, s}(I)}=\inf \left\{\|\widetilde{u}\|_{Z_{b, s}} \mid \widetilde{u}=u \text { on } \mathbb{T} \times I\right\}\right) .
\end{aligned}
$$

For simplicity, we denote $X_{b, s}(I)$ (resp. $\left.Z_{b, s}(I)\right)$ by $X_{s, b}^{T}$ (resp. $\left.Z_{s, b}^{T}\right)$ if $I=(0, T)$. In addition, let

$$
\|u\|_{\mathbb{Z}_{\frac{1}{2}, s}^{T}}:=Z_{\frac{1}{2}, s}^{T} \cap C\left([0, T] ; H^{s}(\mathbb{T})\right) .
$$

There are a series of smoothing estimates:

Lemma 3.1. Let $b, s \in \mathbb{R}$ and $T>0$ be given. There exists a constant $C>0$ such that

(i) for any $\phi \in H^{s}(\mathbb{T})$,

$$
\|W(t) \phi\|_{\mathbb{Z}_{\frac{1}{2}, s}^{T}} \leq\|\phi\|_{s}
$$

(ii) for any $f \in Z_{-\frac{1}{2}, s^{\prime}}^{T}$

$$
\left\|\int_{0}^{t} W(t-\tau) f(\tau) \mathrm{d} \tau\right\|_{\mathbb{Z}_{\frac{1}{2}, s}^{T}} \leq C\|f\|_{Z_{-\frac{1}{2}, s}^{T}} .
$$

Proof. See $[2,9]$. 
Lemma 3.2. For

$$
s \geq s_{0}= \begin{cases}-\frac{1}{2}, & \text { for } l=1 \\ -2, & \text { for } l=2 \\ -\frac{l}{2}, & \text { for } l>2 .\end{cases}
$$

There exist a constant $C$ such that the following bilinear estimate

$$
\left\|(u v)_{x}\right\|_{Z_{-\frac{1}{2}, s}^{T}} \leq C\|u\|_{Z_{\frac{1}{2}, s}^{T}}\|v\|_{Z_{\frac{1}{2}, s}^{T}}
$$

holds.

Proof. See $[17,18]$ and [9].

Proof of Theorem 1.1. Rewrite the system (3.1) in its equivalent integral equation form:

$$
u(t)=W(t) u_{0}+\int_{0}^{t} W(t-\tau)(G h)(\tau) \mathrm{d} \tau-\int_{0}^{t} W(t-\tau)\left(u u_{x}\right)(\tau) \mathrm{d} \tau .
$$

Define

$$
\omega(T, u):=\int_{0}^{T} W(T-\tau)\left(u u_{x}\right)(\tau) \mathrm{d} \tau .
$$

According to Theorem 2.1, for given $u_{0}, u_{1} \in H_{0}^{s}(\mathbb{T})$, if one chooses

$$
h=\Phi\left(u_{0}, u_{1}+\omega(T, u)\right)
$$

in the equation (3.2), then

$$
u(t)=W(t) u_{0}+\int_{0}^{t} W(t-\tau)\left(G \Phi\left(u_{0}, u_{1}+\omega(T, u)\right)\right)(\tau) \mathrm{d} \tau-\int_{0}^{t} W(t-\tau)\left(u u_{x}\right)(\tau) \mathrm{d} \tau
$$

and

$$
\left.u\right|_{t=0}=u_{0},\left.\quad u\right|_{t=T}=u_{1} .
$$

This leads us to consider the map

$$
\Gamma u(t)=W(t) u_{0}+\int_{0}^{t} W(t-\tau)\left(G \Phi\left(u_{0}, u_{1}+\omega(T, u)\right)\right)(\tau) \mathrm{d} \tau-\int_{0}^{t} W(t-\tau)\left(u u_{x}\right)(\tau) \mathrm{d} \tau .
$$

If we can prove that $\Gamma$ is a contraction mapping in an appropriate space, then its fixed point $u$ is a solution of (3.2) with $h=\Phi\left(u_{0}, u_{1}+\omega(T, u)\right)$ and satisfies $\left.u\right|_{t=T}=u_{1}$.

Applying Lemma 3.1-3.2 yields that

$$
\|\Gamma u\|_{\mathbb{Z}_{\frac{1}{2}, s}^{T}} \leq C\left\|u_{0}\right\|_{s}+C\left\|\int_{0}^{t} W(t-\tau)\left(G \Phi\left(u_{0}, u_{1}+w(T, u)\right)\right)(\tau) \mathrm{d} \tau\right\|_{Z_{\frac{1}{2}, s}^{T}}+C\left\|u u_{x}\right\|_{Z_{-\frac{1}{2}, s}^{T}}
$$




$$
\begin{aligned}
& \leq C\left\|u_{0}\right\|_{s}+C\left\|G \Phi\left(u_{0}, u_{1}+\omega(T, u)\right)\right\|_{L^{2}\left([0, T] ; H_{0}^{s}(\mathbb{T})\right)}+C\|u\|_{\mathbb{Z}_{\frac{1}{2}, s}^{T}}^{2} \\
& \leq C\left\|u_{0}\right\|_{s}+C\left[\left\|u_{1}\right\|_{s}+\left\|\left.u_{0}\right|_{s}+\right\| \omega(T, u) \|_{s}\right]+C\|u\|_{\mathbb{Z}_{\frac{1}{2}, s}^{T}}^{2} \cdot
\end{aligned}
$$

Notice that

$$
\begin{gathered}
\quad\|w(T, u)\|_{s}=\left\|\int_{0}^{T} W(T-\tau) u u_{x}(\tau) \mathrm{d} \tau\right\|_{s} \\
\leq C \sup _{t \in(0, T)}\left\|\int_{0}^{t} \psi(t) W(t-\tau) u u_{x}(\tau) \mathrm{d} \tau\right\|_{s} \leq C\|u\|_{\mathbb{Z}_{\frac{1}{2}, s}^{T}}^{2} .
\end{gathered}
$$

Consequently,

$$
\|\Gamma(u)\|_{\mathbb{Z}_{\frac{1}{2}, s}^{T}} \leq C\left(\left\|u_{0}\right\|_{s}+\left\|u_{1}\right\|_{s}\right)+C\|u\|_{\mathbb{Z}_{\frac{1}{2}, s}^{T}}^{2}
$$

For $R>0$, let $B_{R}$ be a bounded subset of $\mathbb{Z}_{\frac{1}{2}, s}^{T}$ :

$$
B_{R}=\left\{v \in \mathbb{Z}_{\frac{1}{2}, s}^{T} \mid[v]=0,\|v\|_{\mathbb{Z}_{\frac{1}{2}, s}^{T}} \leq R\right\}
$$

Then, for any $u \in B_{R}$

$$
\|\Gamma(u)\|_{\mathbb{Z}_{\frac{1}{2}, s}^{T}} \leq C\left(\left\|u_{0}\right\|_{s}+\left\|u_{1}\right\|_{s}\right)+C R^{2} .
$$

We choose $\delta>0$ and $R>0$ such that

$$
2 C \delta+C R^{2} \leq R, \quad C R<\frac{1}{2} .
$$

Then,

$$
\|\Gamma(u)\|_{\mathbb{Z}_{\frac{1}{2}, s}^{T}} \leq R,
$$

which means that $\Gamma$ map $B_{R}$ into itself.

Similarly, for any $u, v \in B_{R}$, we deduce that

$$
\|\Gamma(u)-\Gamma(v)\|_{\mathbb{Z}_{\frac{1}{2}, s}^{T}} \leq \frac{1}{2}\|u-v\|_{\mathbb{Z}_{\frac{1}{2}, s}^{T}}
$$

which implies that $\Gamma$ is an contracting map on $B_{R}$.

By the Banach fixed point theorem, there is unique solution to the integral equation (3.2) which is the desired solution of (3.1). 


\section{Exponential stabilizability}

For the linearized system

$$
\left\{\begin{array}{l}
\partial_{t} u+(-1)^{l+1} \partial_{x}^{2 l+1} u+2 a u_{x}=-K_{\lambda} u, \quad x \in \mathbb{T}, t>0, \\
u(x, 0)=u_{0}(x), \quad x \in \mathbb{T} .
\end{array}\right.
$$

Its solution can be written as

$$
u(t)=W_{\lambda}(t) u_{0},
$$

where $W_{\lambda}$ is the $C_{0}$-semigroup associated to the linearized system.

Lemma 4.1. Let $s \in \mathbb{R}$ and $T>0$ be given. There exists a constant $C>0$ such that

(i)

$$
\left\|W_{\lambda}(t) \phi\right\|_{\mathbb{Z}_{\frac{1}{2}, s}^{T}} \leq C\|\phi\|_{s}
$$

for any $\phi \in H_{0}^{s}(\mathbb{T})$;

(ii)

$$
\left\|\int_{0}^{t} W_{\lambda}(t-\tau) f(\tau) \mathrm{d} \tau\right\|_{\mathbb{Z}_{\frac{1}{2}, s}^{T}} \leq C\|f\|_{Z_{-\frac{1}{2}, s}^{T}}
$$

for any $f \in Z_{-\frac{1}{2}, s}^{T}$.

Proof. For given $\phi \in H_{0}^{s}(\mathbb{T})$ and $f \in Z_{-\frac{1}{2}, s^{\prime}}^{T}$ let

$$
u(t)=W_{\lambda}(t) \phi+\int_{0}^{t} W_{\lambda}(t-\tau) f(\tau) \mathrm{d} \tau .
$$

Then $u$ solves

$$
\left\{\begin{array}{l}
\partial_{t} u+(-1)^{l+1} \partial_{x}^{2 l+1} u=-K_{\lambda} u+f, \quad x \in \mathbb{T}, t>0, \\
u(x, 0)=\phi(x), x \in \mathbb{T} .
\end{array}\right.
$$

Consequently,

$$
u(t)=W(t) \phi+\int_{0}^{t} W(t-\tau) f(\tau) \mathrm{d} \tau-\int_{0}^{t} W(t-\tau)\left[K_{\lambda} u\right](\tau) \mathrm{d} \tau
$$

and for any $0<T^{\prime} \leq T$,

$$
\|u\|_{\mathbb{Z}_{\frac{1}{2}, s}^{T}} \leq C\left(\|\phi\|_{s}+\|f\|_{Z_{-\frac{1}{2}, s}^{T}}\right)+C\left\|K_{\lambda} u\right\|_{Z_{-\frac{1}{2}, s}^{T^{\prime}}}
$$

where $C>0$ depends only on $s$ and $T$. As

$$
\left\|K_{\lambda} u\right\|_{Z_{-\frac{1}{2}, s}^{T^{\prime}}} \leq C_{1}\left\|K_{\lambda} u\right\|_{X_{-\frac{1}{2}+\epsilon, s}^{T^{\prime}}} \leq C_{1}\left(T^{\prime}\right)^{v}\|u\|_{Z_{\frac{1}{2}, s}^{T}}
$$


for some $v>0$ and $C_{1}$ depending only on $s$ and $T$. Thus if $T^{\prime}$ is chosen small enough, we have

$$
\|u\|_{\mathbb{Z}_{\frac{1}{2}, s}^{T}} \leq C\left(\|\phi\|_{s}+\|f\|_{Z_{-\frac{1}{2}, s}^{T}}\right) .
$$

It then follows from the semigroup property of the system (4.1) that

$$
\|u\|_{\mathbb{Z}_{\frac{1}{2}, s}^{T}} \leq C\left(\|\phi\|_{s}+\|f\|_{Z_{-\frac{1}{2}, s}^{T}}\right) .
$$

The proof is complete.

We first show the closed loop system

$$
\left\{\begin{array}{l}
\partial_{t} u+(-1)^{l+1} \partial_{x}^{2 l+1} u+u \partial_{x} u=-K_{\lambda} u, \quad x \in \mathbb{T}, t>0, \\
u(x, 0)=u_{0}(x), \quad x \in \mathbb{T} .
\end{array}\right.
$$

is well-posedness in the space $H^{s}(\mathbb{T})$ for any $s \geq 0$.

Proposition 4.1. Let $T>0$ and $s \geq s_{0}$ be given. Then there exists $a \delta>0$ such that for any $u_{0} \in H^{s}(\mathbb{T})$ with

$$
\left\|u_{0}\right\|_{s} \leq \delta,
$$

the system (4.2) admits a unique solution $u \in \mathbb{Z}_{\frac{1}{2}, s}^{T}$. Moreover, the corresponding solution map is Lipschitz continuous.

Proof. Rewrite the system (4.2) in its equivalent integral equation form:

$$
u(t)=W_{\lambda}(t) u_{0}-\int_{0}^{t} W_{\lambda}(t-\tau)\left(u u_{x}\right)(\tau) \mathrm{d} \tau
$$

Then define the map

$$
\Gamma u(t)=W_{\lambda}(t) u_{0}-\int_{0}^{t} W_{\lambda}(t-\tau)\left(u u_{x}\right)(\tau) \mathrm{d} \tau .
$$

Applying Lemma 4.1, Lemmas 3.1-3.2, we obtain

$$
\begin{aligned}
\|\Gamma u\|_{\mathbb{Z}_{\frac{1}{2}, s}^{T}} & \leq C\left\|u_{0}\right\|_{s}+\left\|\int_{0}^{t}\left[W_{\lambda}(t-\tau)\left(u^{2}\right)_{x}\right](\tau) \mathrm{d} \tau\right\|_{\mathbb{Z}_{\frac{1}{2}, s}^{T}} \\
& \leq C\left\|u_{0}\right\|_{s}+C\|u\|_{\mathbb{Z}_{\frac{1}{2}, s}^{T}}^{2} .
\end{aligned}
$$

For $R>0$, let $B_{R}$ be a bounded subset of $Z_{\frac{1}{2}, s}^{T}$ :

$$
B_{R}=\left\{v \in Z_{\frac{1}{2}, s}^{T} \mid[v]=0,\|v\|_{\mathbb{Z}_{\frac{1}{2}, s}^{T}} \leq R\right\} .
$$


Then, for any $u \in B_{R}$, we have

$$
\|\Gamma(u)\|_{\mathbb{Z}_{\frac{1}{2}, s}^{T}} \leq C\left\|u_{0}\right\|_{s}+C R^{2} .
$$

We choose $\delta>0$ and $R>0$ such that

$$
C \delta+C R^{2} \leq R, \quad C R<\frac{1}{2} .
$$

Then, $\|\Gamma(u)\|_{\mathbb{Z}_{\frac{1}{2}, s}^{T}} \leq R$, which implies that $\Gamma$ maps $B_{R}$ into itself. In addition, for any $u$, $v \in B_{R}$, we have

$$
\|\Gamma(u)-\Gamma(v)\|_{\mathbb{Z}_{\frac{1}{2}, s}^{T}} \leq \frac{1}{2}\|u-v\|_{\mathbb{Z}_{\frac{1}{2}, s}^{T}}
$$

which implies that $\Gamma$ is an contracting mapping on $B_{R}$. By Banach's contracting mapping principle, $\Gamma$ has unique fixed point which is the desired solution of the system (4.2).

Remark 4.1. The local well-posedness result presented in Proposition 4.2 can be restated as follows:

Let $s \geq s_{0}$ and $r>0$ be given. There exists a $T>0$ such that for any $u_{0} \in H^{s}(\mathbb{T})$ with $\left\|u_{0}\right\|_{s} \leq r$, the system (4.2) admits a unique solution $u \in \mathbb{Z}_{\frac{1}{2}, s}^{T}$.

Next we show that the system (4.2) is globally well-posed in the space $H^{s}(\mathbb{T})$ for any $s \geq 0$.

Theorem 4.1. Let $s \geq s_{0}$ and $T>0$ be given. For any $u_{0} \in H^{s}(\mathbb{T})$, the system (4.2) admits a unique solution $u \in \mathbb{Z}_{\frac{1}{2}, s}^{T}$. Furthermore, the following estimate holds

$$
\|u\|_{\mathbb{Z}_{\frac{1}{2}, s}^{T}} \leq \alpha_{T, s}\left(\|u\|_{0}\right)\left\|u_{0}\right\|_{s}
$$

where $\alpha_{T, s}: \mathbb{R}^{+} \rightarrow \mathbb{R}^{+}$is a nondecreasing continuous function depending only on $T$ and $s$.

Proof. The proof is very much similar to that of Theorem 4.7 in [5] and is therefore omitted.

Proof of Theorem 1.2. For given $s \geq 0$ and $\lambda>0$, by Proposition 2.3, there exists positive constant $C$ such that

$$
\left\|W_{\lambda}(t) u_{0}\right\|_{s} \leq C e^{-\lambda t}\left\|u_{0}\right\|_{s}, \quad \forall t \geq 0 .
$$

For any given $0<\lambda^{\prime}<\lambda$, pick $T>0$ such that

$$
2 C e^{-\lambda T} \leq e^{-\lambda^{\prime} T} .
$$


We seek a solution $u$ to the integral equation (4.3) as a fixed point of the map

$$
\Gamma u(t)=W_{\lambda}(t) u_{0}-\int_{0}^{t} W_{\lambda}(t-\tau)\left(u u_{x}\right)(\tau) \mathrm{d} \tau
$$

in some closed ball $B_{R}(0)$ in the function space $\mathbb{Z}_{\frac{1}{2}, s}^{T}$. This will be done provided that $\|\left. u_{0}\right|_{s} \leq \delta$ where $\delta$ is a small number to be determined. Furthermore, to ensure the exponential stability with the claimed decay rate, the numbers $\delta$ and $R$ will be chosen in such a way that

$$
\|u(T)\|_{s} \leq e^{-\lambda^{\prime} T}\left\|u_{0}\right\|_{s} .
$$

By Lemma 4.1, there exist some positive constant $C_{1}, C_{2}$ (independent of $\delta$ and $R$ ) such that

$$
\begin{aligned}
& \|\Gamma(u)\|_{\mathbb{Z}_{\frac{1}{2}, s}^{T}} \leq C_{1}\left\|u_{0}\right\|_{s}+C_{2}\|u\|_{\mathbb{Z}_{\frac{1}{2}, s}^{T}}^{2}, \\
& \left\|\Gamma\left(u_{1}\right)-\Gamma\left(u_{2}\right)\right\|_{\mathbb{Z}_{\frac{1}{2}, s}^{T}} \leq C_{2}\left(\left\|u_{1}\right\|_{\mathbb{Z}_{\frac{1}{2}, s}^{T}}+\left\|u_{2}\right\|_{\mathbb{Z}_{\frac{1}{2}, s}^{T}}\right)\left\|u_{1}-u_{2}\right\|_{\mathbb{Z}_{\frac{1}{2}, s}^{T}} .
\end{aligned}
$$

On the other hand, we have for some constant $C>0$ and all $u \in B_{R}(0)$

$$
\|\Gamma(u)(T)\|_{s} \leq C_{1}\left\|W_{\lambda}(T) u_{0}\right\|_{s}+C_{2}\left\|\int_{0}^{T} W_{\lambda}(T-\tau)\left(u u_{x}\right)(\tau) \mathrm{d} \tau\right\|_{s} \leq e^{-\lambda T} \delta+C^{\prime} R^{2} .
$$

Pick $\delta=C_{4} R^{2}$, where $C_{4}$ and $R$ are chosen so that

$$
\frac{C^{\prime}}{C_{4}} \leq C e^{-\lambda T}, \quad\left(C_{1} C_{4}+C_{2}\right) R^{2} \leq R, \quad 2 C_{2} R \leq \frac{1}{2}
$$

Then we have

$$
\begin{aligned}
& \|\Gamma(u)\|_{\mathbb{Z}_{\frac{1}{2}, s}^{T}} \leq R, \quad \forall u \in B_{R}(0), \\
& \left\|\Gamma\left(u_{1}\right)-\Gamma\left(u_{2}\right)\right\|_{\mathbb{Z}_{\frac{1}{2}, s}^{T}} \leq \frac{1}{2}\left\|u_{1}-u_{2}\right\|_{\mathbb{Z}_{\frac{1}{2}, s}^{T}}, \quad \forall u_{1}, u_{2} \in B_{R}(0) .
\end{aligned}
$$

Therefore, $\Gamma$ is a contraction in $B_{R}(0)$. Furthermore, its unique fixed point $u \in B_{R}(0)$ fulfills

$$
\|u(T)\|_{s} \leq\|\Gamma(u)(T)\|_{s} \leq e^{-\lambda^{\prime} T} \delta .
$$

Assume now that $0<\left\|u_{0}\right\|_{0)}<\delta$. Changing $\delta$ into $\delta^{\prime} \equiv\left\|u_{0}\right\|_{s}$ and $R$ into $R^{\prime} \equiv\left(\delta^{\prime} / \delta\right)^{\frac{1}{2}} R$, we infer that

$$
\|u(T)\|_{s} \leq e^{-\lambda^{\prime} T}\left\|u_{0}\right\|_{s}
$$

and an obvious induction yields

$$
\|u(n T)\|_{s} \leq e^{-\lambda^{\prime} n T}\left\|u_{0}\right\|_{s}
$$


for any $n \geq 0$. We infer by the semigroup property that there exists some positive constant $C>0$ such that

$$
\|u(t)\|_{s} \leq C e^{-\lambda^{\prime} t}\left\|u_{0}\right\|_{s}
$$

if $\left\|u_{0}\right\|_{s} \leq \delta$.

\section{Acknowledgement}

The first author is financially supported by the Natural Science Foundation of Zhejiang Province (\# LY18A010024, \# LQ16A010003), the China National Natural Science Foundation (\# 11505154, \# 11605156) and the Open Foundation from Marine Sciences in the Most Important Subjects of Zhejiang (\# 20160101). The second author is financially supported by Foundation for Distinguished Young Teacher in Higher Education of Guangdong, China (YQ2015167), Foundation for Characteristic Innovation in Higher Education of Guangdong, China (Analysis of some kinds of models of cell division and the spread of epidemics), NSF of Guangdong Province (2015A030313707). The authors are greatly in debt to the anonymous referee for his/her valuable comments and suggestions on modifying this manuscript.

\section{References}

[1] Gorsky J., Himonasb A., Well-posedness of KdV with higher dispersion. Mathematics and Computers in Simulation, 80 (2009) 173-183.

[2] Hirayama H., Local well-posedness for the periodic higher order KdV type equations. NoDEA Nonlinear Differential Equations, DOI 10.1007/s00030-011-0147-9.

[3] Wazwaz A.M., Partial equations and solitary waves theory-Nonlinear Physical Science Series, Springer Dordrecht Heidelberg London New York, 2009.

[4] Laurent C., Rosier L., Zhang B. Y., Control and stabilization of the Korteweg-de Vries equation on a periodic domain. Communications in Partial Differential Equations, 35 (2010), 707-744.

[5] Russell D. L., Zhang B.-Y., Exact controllability and stabiability of the Korteweg-de Veies equation, Transaction of the American Mathematical Society. 384 (1996), 3643-3672.

[6] Russell D. L., Zhang B.-Y., Controllability and stabilizability of the third-order linear dispersion equation on a periodic domain. SIAM J. Control and Optimization,31 (1993), 659-676.

[7] Zhang B.-Y., Zhao X. Q., Control and stabilization of the Kawahara equation on a periodic domain. Communications in Information and Systems, 12 (2012), 77-96.

[8] Zhao X. Q., Zhang B.-Y., Global controllability and stabilizability of the Kawaraha equation on a periodic domain, to appear.

[9] Li J. F., Shi S. G., Local well-posedness for the dispersion generalized periodic KdV equation. J. Math. Anal. Appl., 379 (2011), 706-718.

[10] Bona J. L., Sun S. M., Zhang B.Y., Boundary smoothing properties of the Korteweg-de Vries equation in a quarter plane and applications. Dyn. Partial Differ. Equ., 3 (2006) 1, 1-69. 
[11] Bona J. L., Sun S. M., Zhang B.Y., Non-homogeneous boundary value problems for the Kortewege Vries and the Korteweg de Vries Burgers equations in aquarter plane. Ann. I. H. Poincaré-AN, 25 (2008), 1145-1185.

[12] Bona J. L., Sun S. M., Zhang B.Y., The Korteweg-de Vries equation on a finite domain II. Journal of Differential Equations, 247 (2009), 2558-2596.

[13] Kuvshinov R. V., Faminskii A. V., Mixed problem for the Kawahara equation in a half-strip. Differential Equations, 45 (2009), 404C415.

[14] Sangare K., Faminskii A. V., Weak solutions of a mixed problem in a half-strip for a generalized kawahara equation. Math. Notes, 85(2009), 90-100.

[15] Pazy A., Semigroups of Linear Operators and Applications to Partial Differential Equations, Appl. Math. Sci., vol. 44, Springer-Verlag, New York, 1983.

[16] Slemrod M., A note on complete controllability and stabilizability for linear control systems in Hilbert space. SIAM J. Control, 12 (1974), 500-508.

[17] Bourgain J., Fourrier transform restriction phenomena for certain lattice subsets and applications to nonlinear evlution equations, Part I: The Schrödinger equation, Part II: The KdV equation. Geom. Funct. Anal., 3 (1993), 107-156, $209-262$.

[18] Kato T., Lower regularity well-posedness for the periodic Kawahara equation, arXiv: 1203.2275v1 [math.AP] 10 Mar 2012.

[19] Tao T., Nonlinear Dispersive Equations, Local and Global Analysis, CBMS Regional Conference Series in Mathematics. Vol. 106., Providence, RI: AMS, 2006.

[20] Tartar L., Nonlinear interpolation and regularity. J. Funct. Anal., 9 (1972), 469-489. 